Curr Opin Hematol. 2006 November ; 13(6): 419-425. doi:10.1097/01.moh.0000245697.54887.6f.

\title{
Mesenchymal Stromal Cells
}

\author{
Edwin M. Horwitz, Michael Andreef, and Francesco Frassoni
}

\section{Introduction}

Mesenchymal stromal cells (MSCs) are the spindle shaped plastic-adherent cells isolated from bone marrow, adipose, and other tissue sources, with multipotent differentiation capacity in vitro. However, whether MSCs truly qualify as stem cells is an area of some debate[1]. MSCs were first described by Friendenstein as hematopoietic supportive cells of bone marrow. He showed that MSCs could differentiate to bone in vitro and a subset of the cells had a high proliferative potential (CFU-F) when plated at low density in tissue culture[2,3]. Based largely on Friendenstein's work, Maureen Owen proposed the existence of a stromal stem cell to maintain the marrow microenvironment as the hematopoietic stem cell maintains hematopoiesis[4]. The notion of a mesenchymal stem cell was popularized by Arnold Caplan proposing that MSCs gave rise to bone, cartilage, tendon, ligament, marrow stroma, adipocytes, dermis, muscle and connective tissue[5]. However, convincing data to support the "stemness" of these cells were not forthcoming, and now most investigators recognize that in vitro isolated MSCs are not a homogenous population of stem cells, although a bona fide mesenchymal stem cell may reside within the adherent cell compartment of marrow[6].

MSCs undoubtedly play a critical role in the marrow microenvironment. Following intramedullary transplantation of eGFP-marked human MSCs into a NOD SCID mouse, the MSCs incorporated into the murine marrow microenvironment and improved the human hematopoietic stem cell activity in the host mouse[7]. MSCs are also thought to be of great value for cell based therapies. This discussion will focus on the properties of MSCs that engender their utility as therapeutic cells and specifically on MSCs as treatment for GVHD and as targeting vehicles for anti-tumor therapies.

\section{Nomenclature}

As stated above, data to support the designation of MSCs as biologically functional stem cells are lacking. However, the acronym, MSC, is firmly engrained in the vernacular of cell biologists and clinical cell therapists. Thus, the International Society for Cellular Therapy (ISCT) has recommended that these spindle-shaped, plastic-adherent cells be termed, "mesenchymal stromal cells" [6]. This label allows investigators to continue to use the acronym, MSCs, which should reduce the potential for confusion in the literature. A biologically active stem cell for mesenchymal tissues may exist, but the term "mesenchymal stem cell" should be reserved for the subset of mesenchymal cells that demonstrate stem cell activity by rigorous criteria.

Publisher's Disclaimer: This is a PDF file of an unedited manuscript that has been accepted for publication. As a service to our customers we are providing this early version of the manuscript. The manuscript will undergo copyediting, typesetting, and review of the resulting proof before it is published in its final citable form. Please note that during the production process errors may be discovered which could affect the content, and all legal disclaimers that apply to the journal pertain. 


\section{Phenotype}

The defining characteristics of MSCs are inconsistent among investigators due, in part, to the lack of a universally accepted surface marker phenotype. However, all proposed MSC populations are plastic adherent in vitro; hence, this is one defining characteristic. The first important studies of surface antigen markers led to the development of $\mathrm{SH} 2$ and $\mathrm{SH} 3$, antibodies which seemed to identify MSCs[8]. Subsequently, SH2 and SH3 were shown to recognize epitopes on CD105 and CD73, respectively[9,10]. Furthermore, CD90 is expressed on all cells that we accept as MSCs. These cells do not express hematopoietic antigens, e.g. CD45, CD34, CD14, CD19, or CD3. Additionally, MSCs express MHC Class I molecules in vitro, but not Class II molecules unless stimulated, e.g. by interferon, in tissue culture. Thus, a surface marker phenotype of MSCs is CD105+, CD73+, CD90+, CD45-, CD34- CD14-, CD19-, CD3-, HLA DR-. While unequivocally identifying MSCs, this surface marker profile is cumbersome. Stable, pancellular expression of surface markers that are unique to MSCs within the bone marrow, the most common source of MSCs, would greatly facilitate the identification of these cells.

The single most characteristic feature of MSCs is the capacity to differentiate to osteoblasts, adipocytes, and chondroblasts in vitro. It is therefore quite reasonable for investigators to demonstrate such trilineage differentiation in vitro to prove their cells under study are MSCs.

In practice, MSCs can be defined by the criteria shown in the Table, as proposed by the ISCT Mesenchymal and Tissue Stem Cell Committee[11]. The criteria are designed not only to define the MSCs, but also to exclude hematopoietic cells, which is important since, as stated above, MSCs are most commonly isolated from bone marrow. CD3 expression is not included in the criteria because T cells are uncommon contaminants of MSC preparations. It is important to avoid hematopoietic cells among the populations of MSCs being used for cell therapy studies because they could alter the scientific outcomes and may be deleterious for patients in clinical trials.

\section{Isolation of MSCs}

For obvious reasons, if the proposed therapeutic cells are not readily accessible, clinical utility is limited. Effective cell therapy, therefore, begins with a cell type that is relatively easy to isolate. MSCs are most often isolated by "adherence selection." For example, bone marrow mononuclear cells are placed in a plastic tissue culture vessel and maintained for 15 days at $37^{\circ} \mathrm{C}$. Then, the nonadherent cells are removed as the media is changed and the remaining adherent cells are isolated MSCs. At this stage, the MSC cultures are definitely not free of contamination by resident tissue cells, e.g. hematopoietic cells; however, successive passages of the ex vivo expanded cells effectively remove most or all contaminating cells. Thus, tissue culture serves to expand and purify the MSCs. Similarly, when other sources of MSCs, e.g. adipose tissue, a mononuclear cell preparation is maintained in tissue culture to isolate the MSCs.

\section{MSCs as Cell Therapy for Tissue Regeneration}

There are three fundamental questions that must be addressed when using MSCs as cell therapy for tissue regeneration. First, will MSCs differentiate to the tissue of interest in vivo? This is a critically important issue as certain culture conditions may induce atypical differentiation in vitro that may not occur in vivo. Additionally, MSCs may not differentiate to the targeted tissue, but instead generate cell types that function in a beneficial way within the tissue. For example MSCs may secrete useful soluble mediators that foster repair of a 
tissue so that differentiation is unneeded for clinical benefit. Thus, MSCs may be highly effective for applications in regenerative medicine by several mechanisms.

Second, how can the cells be delivered to the relevant tissue(s)? For example, if intravenously infused, will MSCs home to the desired sites? Although some investigators have suggested that MSCs home to sites of inflammation, it is unclear that MSCs home to sites of other types of local or systemic disease, and there is little data indicating that MSCs home to healthy tissue. Despite the uncertainty of homing to diseased tissues, sufficient intravenously infused MSCs may arrive and incorporate in the desired tissue to generate clinical benefits. For example, Horwitz et al. reported the infusion of MSCs after BMT into children with osteogenesis imperfecta, a metabolic bone disorder. Engraftment and growth acceleration was demonstrated in 5 of 6 patients[12]. Koc et al. reported MSC infusion in children with metachromatic leukodystrophy and Hurler's disease after BMT. In 4 of 6 patients with metachromatic leukodystrophy, an improvement in nerve conduction velocity was observed, but engraftment in the neural tissue was not assessed[13]. In both cases, homing strictly defined was not demonstrated; however the former study showed the presence of intravenously infused cells within the targeted tissue.

Third, how much tissue replacement by donor cells (i.e. engraftment) is needed to achieve correction or improvement of the damage or diseased tissue? The answer will likely be tissue and disease specific, and therefore will require animal models that reliably model the human disease, or more effectively, pilot clinical trials. Importantly, the level of tissue replacement is often quite low, far less than what may be hypothesized; consequently, estimates are useful to determine which diseases should be investigated, but experimental data are essential to formulate therapeutic strategies.

\section{Immunologic properties of MSCs}

Any cell employed for therapeutic purposes would ideally be immunoprivileged allowing for use in HLA mismatched patients. Further, cells that can regulate the immune response could be effectively used to modulate the immune system to treat immunologic disease. MSCs have been reported to be immunosuppressive and immunoprivileged. The two terms are often used interchangeably; however, this is strictly incorrect. A cell may escape immune recognition (i.e. immunoprivileged) without having an effect on immune effector cells. Similarly, a cell may secrete immunosuppressive molecules while being recognized by an allogeneic immune system. MSCs do seem to exhibit an effect on the immune effector cells in vitro. This property has led to much dialogue whether MSCs could be effective therapy for autoimmune diseases such as rheumatoid arthritis. More important for this discussion is the role of MSCs in the treatment Graft-versus-Host Disease (GVHD).

\section{Mesenchymal Stem Cells for the Treatment of Graft-versus-Host Disease}

As mentioned above, MSCs are an essential component of the stromal scaffold of the bone marrow that provides physical and functional support during hematopoiesis. Based on this concept, MSCs have been studied for their ability to improve engraftment of hematopoietic stem cells in vivo[14,15]. While some reports suggest that MSCs increase engraftment, the data are not particularly impressive, at least in the models utilized. It has been recently shown that MSCs exert a profound immunomodulatory effect by means of both soluble and cell contact-dependent mechanisms[16]. MSCs can act both on T and B cells and although several mechanisms of action have been suggested, the data are contradictory. The ability to inhibit or stimulate T-cell alloresponses appears to be independent of HLA matching. It is still unclear whether MSCs naturally exhibit an immunoregulatory role or whether this is the consequence of a more general, non-specific interference with the cell cycle[17]. 
In this context, it is interesting to note that stromal cells, together with osteoblasts and endothelial cells, contribute to the formation of the HSC niche. This can be defined as a specialized microenvironment that precisely maintains a long-term storage of quiescent, slowly dividing HSCs by preventing their proliferation, differentiation or apoptosis. It can be hypothesized that MSCs, on one hand, are preventing T lymphocyte activation and proliferation (to prevent possible harm on HSC) and, on the other hand, seem to exert a potent anti-apoptotic effect. Although the mechanisms of immunomodulation are still unfolding, a relevant in vivo immunomodulatory effect has been shown: 1) if given in patients with severe acute GVHD, they are able to reverse the evolution of GVHD in a significant proportion of patients[18,19], and 2) in a recent in vivo experiment in which injection of MSCs ameliorated the course of chronic progressive experimental autoimmune encephalomyelitis (EAE), the mouse model of multiple sclerosis[20].

The EBMT MSC Expansion Consortium used MSCs to treat grades III-IV GVHD in 40 patients who were resistant to second line GVHD treatment. The MSC dose was a median $1.0 \times 10^{6}$ cells $/ \mathrm{kg}$ recipient body weight (range $0.4-9 \times 10^{6}$ cells $/ \mathrm{kg}$ ). Adverse effects were not seen after MSC infusions. Nineteen patients received one dose, 19 patients received 2 doses, one patient received 3 doses, and one patient received 5 doses. In some cases, an individual patient received MSC doses from different donors. The MSC donors were HLAidentical siblings in 5 cases, haploidentical donors in 19 cases, and 41 cases of third-party HLA-mismatched donors. Among the 40 patients treated for severe acute GVHD, 19 had complete responses, 9 showed improvement, 7 did not respond, 4 had stable disease and 1 was not evaluated due to short follow-up. Ectopic tissue formation was not seen. MSC dramatically affected tissue repair of severe acute GVHD of the gut, liver, and skin in a consistent proportion of patients. Twenty-one patients are alive with between 6 weeks and 3.5 years follow-up after transplantation. Nine of these patients have extensive chronic GVHD. One patient with ALL has recurrent leukemia and one patient has de novo AML of host origin. In view of the dismal outcome in patients with grades III-IV acute GVHD, the data from this small trial are promising. However, the optimal strategy for the treatment of GVHD based on MSC infusion has not yet been determined and remains rather complex for a several reasons: 1) the ex vivo cell expansion is expensive and time consuming; 2) there is variation in the expansion capability from donor to donor; 3 ) often, previously expanded MSCs are required for the timely treatment of GVHD; 4) the optimal dose of MSCs, or the need for multiple infusions, to obtain the maximal effect on GVHD is unknown; 5) expanded MSCs are very difficult to detect after infusion, and the patients' marrow stroma remain of host origin with the possible exception of some pediatric patients.

Ongoing efforts within the EBMT Consortium are addressing these challenges in an effort to determine the role of MSC therapy in the treatment for GVHD. At the current state of research, we conclude that MSCs have both immunomodulatory and tissue repairing effects and should be further explored as treatment of severe acute GVHD in prospective randomized trials.

\section{MSCs as Tumor-Targeting Drug Delivery Systems}

The formation of stroma is essential for tumor growth and involves complex interactions between malignant tumor cells and non-tumor stromal cells. Studeny et al. have demonstrated that MSCs integrate into solid tumors, suggesting the development of anticancer therapies based on the intratumoral production of agents by gene-modified MSCs[21-23].

Andreeff and colleagues have now conducted a series of experiments to address this issue by noninvasively visualizing MSCs using luciferase bioluminescence. The cells were labeled 
by a fiber modified adenoviral vector expressing firefly luciferase (AdLux-F/RGD) and the MSC-Lux were injected into normal (healthy) SCID mice or mice bearing established metastatic breast or ovarian tumors. Biodistributed MSC-Lux were imaged utilizing the Xenogen IVIS detection system. In normal mice, human MSC (hMSC) migrated to the lungs where they remained resident for 7-10 days. In animals bearing established metastatic lung tumors, IV injected hMSC again migrated to the lungs. However, in contrast to control mice, the Lux signal remained strong over a 15-day period with only a slight decrease over the first 10 days. After IP injection, hMSC-LUX were detected in the peritoneum, and after 7 days, no hMSC-LUX was detected in normal animals, while strong punctate regions of LUX-activity were observed in ovarian tumors. In contrast to SCID mice injected with hMSC, when healthy Balb/C mice were injected, Balb/C derived MSC-LUX initially migrated to the lungs, but within $2.5 \mathrm{hrs}$ had exited the lungs to remain liver and spleen resident for 5-7 days. Tumor cells were then transduced with renilla luciferase constructs allowing for the co-localization and dynamic interactions of firefly luciferase MSCs and renilla luciferase tumors to be demonstrated.

hMSC-producing interferon-beta (IFNb-MSC) were found to inhibit the growth of metastatic tumors in the lungs of SCID mice. When injected IV (4 doses of $10^{6} \mathrm{MSC} /$ week) into SCID mice bearing pulmonary metastases of carcinomas or melanomas, tumor growth was significantly inhibited as compared to untreated or vector-control MSC controls ( $\mathrm{p}=$ 0.007), while recombinant IFNb protein (50,000 IU qod) was ineffective ( $\mathrm{p}=0.14)$. IV injected IFNb-MSC prolonged the survival of mice bearing metastatic breast carcinomas $(\mathrm{p}=0.001)$. Intraperitoneal (IP) injections of IFN-MSC into mice carrying ovarian carcinomas resulted in doubling of survival in SKOV-3, and cures in $70 \%$ of mice carrying OVAR-3 tumors.

A similar strategy is also effective as therapy for brain tumors. MSC injected into the ipsilateral or contralateral carotid artery were found to localize to glioma xenografts in mice and IFNb-MSC significantly $(\mathrm{p}<0.05)$ prolonged survival of these mice[24].

These data suggest that systemically administered gene-modified MSC selectively engrafts into the tumor microenvironment and remain resident as part of the tumor architecture. MSC-expressing IFN-b inhibit the growth of melanomas, gliomas, metastatic breast and ovarian cancers in vivo and prolong the survival of mice bearing established tumors. Thus, MSCs are potentially a universal vehicle to deliver localized antitumor therapy. Clinical trials, which are in development, will be conducted to test these experimental findings.

\section{Conclusions}

MSCs have an enormous potential as cell therapy in tissue regeneration, immune modulation, and as delivery vehicles for the specific delivery vehicles for anti-tumor agents, but the true clinical utility remains to be proven. MSCs are relatively easy to isolate and purify, and we currently have means to unequivocally identify the cells, although more specific surface markers are needed. MSCs have been infused into well over a hundred patients, including young children, without serious adverse events testifying to the general safety of this strategy. Future efforts in our field must focus on better defining the therapeutic potential of MSCs through clinical trials and better understanding of the biology of MSCs to elucidate the mechanisms of these therapeutic effects.

\section{References}

1. Horwitz EM, Keating A. Nonhematopoietic mesenchymal stem cells: what are they? Cytotherapy. 2000; 2:387-388. [PubMed: 12044232] 
2. Friedenstein AJ, Petrakova KV, Kurolesova AI, Frolova GP. Heterotopic of bone marrow. Analysis of precursor cells for osteogenic and hematopoietic tissues. Transplantation. 1968; 6:230-247. [PubMed: 5654088]

3. Friedenstein AJ, Deriglasova UF, Kulagina NN, et al. Precursors for fibroblasts in different populations of hematopoietic cells as detected by the in vitro colony assay method. Exp. Hematol. 1974; 2:83-92.

4. Owen M, Friedenstein AJ. Stromal stem cells: marrow-derived osteogenic precursors. Ciba Found Symp. 1988; 136:42-60. [PubMed: 3068016]

5. Caplan AI. Mesenchymal stem cells. J Orthop Res. 1991; 9:641-650. [PubMed: 1870029]

6. Horwitz E, Le Blanc K, Dominici M, et al. Clarification of the nomenclature for MSC: The International Society for Cellular Therapy position statement. Cytotherapy. 2005; 7:393-395. [PubMed: 16236628]

7. Muguruma Y, Yahata T, Miyatake H, et al. Reconstitution of the functional human hematopoietic microenvironment derived from human mesenchymal stem cells in the murine bone marrow compartment. Blood. 2006; 107:1878-1887. [PubMed: 16282345]

8. Pittenger MF, Mackay AM, Beck SC, et al. Multilineage potential of adult human mesenchymal stem cells. Science. 1999; 284:143-147. [PubMed: 10102814]

9. Barry FP, Boynton RE, Haynesworth S, Murphy JM, Zaia J. The monoclonal antibody SH-2, raised against human mesenchymal stem cells, recognizes an epitope on endoglin (CD105). Biochem Biophys Res Commun. 1999; 265:134-139. [PubMed: 10548503]

10. Barry F, Boynton R, Murphy M, Zaia J. The SH-3 and SH-4 Antibodies Recognize Distinct Epitopes on CD73 from Human Mesenchymal Stem Cells. Biochem Biophys Res Commun. 2001; 289:519-524. [PubMed: 11716504]

11. Dominici M, Le Blanc K, Mueller I, et al. Minimal criteria for defining multipotent mesenchymal stromal cells. The International Society for Cellular Therapy position statement. Cytotherapy. 2006; 8:315-317. [PubMed: 16923606]

12. Horwitz EM, Gordon PL, Koo WKK, Marx JC, Neel MD, McNall RY, Muul L, Hofmann T. Isolated allogeneic bone marrow-derived mesenchymal cells engraft and stimulate growth in children with osteogenesis imperfecta: implications for cell therapy of bone. Proc Natl Acad Sci USA. 99:8932-8937.

13. Koc ON, Day J, Nieder M, et al. Allogeneic mesenchymal stem cell infusion for treatment of metachromatic leukodystrophy (MLD) and Hurler syndrome (MPS-IH). Bone Marrow Transplant. 2002; 30:215-222. [PubMed: 12203137]

14. Koc ON, Gerson SL, Cooper BW, et al. Rapid hematopoietic recovery after coinfusion of autologous-blood stem cells and culture-expanded marrow mesenchymal stem cells in advanced breast cancer patients receiving high-dose chemotherapy. J Clin Oncol. 2000; 18:307-316. [PubMed: 10637244]

15. Lazarus HM, Koc ON, Devine SM, et al. Cotransplantation of HLA-identical sibling cultureexpanded mesenchymal stem cells and hematopoietic stem cells in hematologic malignancy patients. Biol Blood Marrow Transplant. 2005; 11:389-398. [PubMed: 15846293]

16. Le Blanc K, Ringden O. Immunobiology of human mesenchymal stem cells and future use in hematopoietic stem cell transplantation. Biol Blood Marrow Transplant. 2005; 11:321-334. [PubMed: 15846285]

17. Glennie S, Soeiro I, Dyson PJ, Lam EW, Dazzi F. Bone marrow mesenchymal stem cells induce division arrest anergy of activated T cells. Blood. 2005; 105:2821-2827. [PubMed: 15591115]

18. Ringden O, Uzunel M, Rasmusson I, et al. Mesenchymal stem cells for treatment of therapyresistant graft-versus-host disease. Transplantation. 2006; 81:1390-1397. [PubMed: 16732175]

19. Le Blanc K, Rasmusson I, Sundberg B, et al. Treatment of severe acute graft-versus-host disease with third party haploidentical mesenchymal stem cells. Lancet. 2004; 363:1439-1441. [PubMed: 15121408]

20. Zappia E, Casazza S, Pedemonte E, et al. Mesenchymal stem cells ameliorate experimental autoimmune encephalomyelitis inducing T-cell anergy. Blood. 2005; 106:1755-1761. [PubMed: 15905186] 
21. Studeny M, Marini FC, Champlin RE, Zompetta C, Fidler IJ, Andreeff M. Bone marrow-derived mesenchymal stem cells as vehicles for interferon-beta delivery into tumors. Cancer Res. 2002; 62:3603-3608. [PubMed: 12097260]

22. Studeny M, Marini FC, Dembinski JL, Zompetta C, Cabreira-Hansen M, Bekele BN, Champlin RE, Andreeff M. Mesenchymal stem cells: Potential precursors for tumor stroma and targeted delivery vehicles of anti-cancer agents. J Natl Cancer Inst. 2004; 96:1593-1603. [PubMed: 15523088]

23. Marini, F.; Hall, B.; Dembinski, J.; Studeny, M.; Sasser, AK.; Andreeff, M. Mesenchymal stem cells as vehicles for genetic targeting of tumors. In: Ho, AD.; Hoffman, R.; Zanjani, ED., editors. Stem Cell Transplantation. Wiley-VCH Verlag; Weinheim, Germany: 2006. p. 157-175.

24. Nakamizo A, Marini F, Studeny M, Gumin J, Chen J, Hentschel S, Vecil G, Dembinski J, Andreeff M, Lang FF. Human bone marrow-derived mesenchymal stem cells in the treatment of gliomas. Cancer Res. 2005; 65:3307-3318. [PubMed: 15833864] 
Table. Summary of Criteria to Identify Mesenchymal Stromal Cells (MSCs).

\begin{tabular}{|c|c|c|}
\hline \multicolumn{3}{|l|}{ 1. Adherence to plastic in standard culture conditions } \\
\hline 2. Phenotype & Positive $(叉 95 \%+)$ & Negative $(<2 \%+)$ \\
\hline & CD105 & CD45 \\
\hline & CD73 & CD34 \\
\hline & CD90 & $\mathrm{CD} 14$ or $\mathrm{CD} 11 \mathrm{~b}$ \\
\hline & & $\mathrm{CD} 79 \mathrm{a}$ or $\mathrm{CD} 19$ \\
\hline & & HLA-DR \\
\hline 3. Differentiation: (by staining of in vitro cell culture) & Bone, Adipose, $\mathrm{Ca}$ & \\
\hline
\end{tabular}

